\title{
Diagnosis of Pulmonary Hypertension Using Ultrasonography in the Management of Critical Patients in the Intensive Care Unit
}

\author{
Eka Adhiany ${ }^{1}$, Muhammad Iqbal ${ }^{2}$ \\ ${ }^{1,2}$ Department of Anesthesiology and Intensive Therapy, Faculty of Medicine, Universitas Syiah Kuala, \\ Dr. Zainoel Abidin Public Hospital, Banda Aceh, Indonesia \\ eadhiany@unsyiah.ac.id
}

\begin{abstract}
Pulmonary arterial hypertension is most often diagnosed in its advanced stages because of the nonspecific nature of early symptoms and signs. Although clinical assessment is essential when evaluating patients with suspected pulmonary arterial hypertension, echocardiography is a key screening tool in the diagnostic algorithm. Echocardiography is valuable in assessing prognosis and treatment options, monitoring the efficacy of specific therapeutic interventions, and detecting the preclinical stages of disease. In this report, we reported A 35 years old woman admitted to the emergency unit with breathing difficulty which has felt since 2 days before. she transferred from rural hospital after hospitalized for a week. The patient has history of spinal surgery due to spondylitis tuberculosis 8 months ago and has an anti-tuberculosis drug for 2 months. The vital signs show respiratory failure with blood pressure $114770 \mathrm{mmHg}$, heart rate 118 bpm, respiratory rate 35-40 bpm and pulse saturation 80-85\% with non-rebreathing mask. Physical examination shows increasing work of breathing, without rales and wheezing, liver enlargement palpated 3 fingers below right ribs the chest $x$ ray shows cardiomegaly and right pleural effusion
\end{abstract}

Keywords: pulmonary; hypertension; ultrasonography

\section{Introduction}

Pulmonary hypertension in daily clinical practice is often diagnosed at an advanced stage because the initial signs and symptoms are not specific. Although clinical assessment is very important in assessing patients who are suspected of having pulmonary hypertension, echocardiography is the main medium of choice in diagnostic enforcement. This examination provides an estimate of the value of the pulmonary artery pressure, both at rest and during exercise, and is also useful in screening for secondary causes of pulmonary hypertension. Echocardiography is also useful in assessing prognosis and treatment options, monitoring the effectiveness of certain therapeutic interventions, and assessing the preclinical stage of the disease.

\section{Case Report}

A 35 year old female patient was referred from the regional hospital after being treated for 1 week with complaints of shortness of breath. Shortness of breath getting worse since 2 days ago. The patient had a history of tuberculous spondylitis and had undergone posterior stabilization surgery 8 months ago. The patient had been taking antituberculosis drugs for the last 2 months. The physical examination revealed signs of respiratory failure. Vital signs show blood pressure $114 / 70 \mathrm{mmHg}$, pulse rate $118 \mathrm{x} /$ minute, breath rate $35-40 \mathrm{x} / \mathrm{min}$ and peripheral saturation $80-85 \%$ using supplemental oxygen rebreathing mask. 
Figure 1. FATE with a Four Chamber Window with a Picture of the Widening of the Right Ventricle (D Shape)

There was an increase in breathing effort, ronkhi and wheezing were not found, the edge of the liver was felt 3 fingers under the right rib. Chest radiograph shows enlarged heart and right pleural effusion. The patient was then intubated and treated in the Intensive Care (ICU) room. Focused assessed transthoracic echocardiography (FATE) was examined. We found enlargement of the inferior diameter of the vena cava (IVC), tricuspid regurgitation with a pressure gradient of $33.5 \mathrm{mmHg}$ and enlargement of the right ventricle with a D-shape image. The patient was diagnosed with right heart failure caused by pulmonary hypertension. Patients are given ventilatory support, inodilators with milrinone titrations ranging from 0.375 to $0.5 \mathrm{mcg} / \mathrm{kg} \mathrm{/} \mathrm{min,} \mathrm{broad} \mathrm{spectrum} \mathrm{antibiotics,}$ thromboprophylaxis and other supportive therapies. On the third day of ICU care,

\section{Discussion}

Pulmonary hypertension (Pulmonary Arterial Hypertension) is a hemodynamic and pathophysiological condition that occurs due to an increase in the mean pulmonary artery pressure (MPAP). $\geq 25 \mathrm{mmHg}$ ) at rest as assessed using a right heart catheter. This condition can be found in several clinical conditions with different pathogenetic and clinical features, such as pulmonary arterial hypertension and left heart disease, pulmonary disease, and thromboembolism.

In particular, pulmonary hypertension is characterized by an increase in precapillary pulmonary artery pressure caused by obstruction of proximal blood flow from the pulmonary capillaries and an increase in pulmonary vascular resistance. This results in an excessive increase in pressure in the right ventricle which can eventually lead to right heart failure and death. Pulmonary hypertension has an incidence prevalence ranging from 30 to 50 cases per one million individuals. It is more common in women than men, and can be idiopathic, inherited, induced by drugs or toxins, or associated with other medical conditions, such as congenital heart disease, connective tissue disease, HIV infection, portal hypertension, schistosomiasis, and chronic hemolytic anemia (Abbas AE, 2003).

Given the non-specific symptoms and signs of pulmonary hypertension, especially in its early stages, a high index of clinical suspicion is required to detect this state before irreversible pathophysiological changes occur. In this regard, transthoracic echocardiography by providing direct or indirect signs of elevated pulmonary artery pressure (Pulmonary Artery Pressure), is an excellent non-invasive screening test for patients with symptoms or risk factors for pulmonary hypertension, such as connective tissue disease, pulmonary embolism, heart failure, and heart murmurs. This examination can also provide important information regarding the etiology and prognosis of pulmonary hypertension (Bossone E, 2013). 
According to Sangy (2020) Hypertension is one of the most common and prevalent diseases in the world today. It often grows without being noticed and it may end up with cardiovascular diseases or severe heart failure. Therefore, hypertension should be controlled and treated carefully. Hypertension is one of the main risk factors for all types of stroke whether it's a bleeding stroke or an infarction stroke. Hypertension is in line with the increased risk of stroke (Pinzon \& Asanti in Sari, 2020). Sorganvi, et al in India in Sari (2020) showed that hypertension increases the risk ofstroke by 3.80 times. High blood pressure plays a role in atherosclerotic reactions through the risk of pressure on endotheliocytes and layers in the lining of artery walls caused by the formation of plaque in a person's blood vessels called high blood pressure when blood pressure $\geq 140 / 90 \mathrm{mmHg}$ (Pinzon \& Asanti in Sari, 2020). Research conducted by (Nastiti in Sari, 2020) states that there is an influence between the occurrence of strokes with high blood pressure and high blood pressure is the most important cause of theeffect on the occurrence of stroke. In general, the common risk factors for stroke include hypertension, diabetes, smoking, obesity, atrial fibrillation, and drug use. Of all the above risk factors, hypertension is the most common modifiable risk factor for stroke (Syahrul, 2020).

Cardiovascular diseases (CVD) are one of the major reasons of death in the whole world. It covers more than one third of death causes in western countries and 50 percent of death causes in Iran which is also the major cause of death. Studies have shown that systematic and chronic inflammation can increase getting cardiovascular disease.Doppler echocardiography examination allows the measurement of an accurate estimate of the pulmonary artery pressure (Pulmonary Artery Pressure), because in the absence of pulmonary flow restriction, the peak velocity (peak velocity) of tricuspid regurgitation (TR) and right ventricular tract acceleration time (RVOT) have a positive linear correlation. and negative, respectively, by pulmonary artery systolic pressure (SPAP) and mean pulmonary artery pressure (MPAP) as measured with a right heart catheter. In addition, the initial diastolic and late diastolic peak velocities of pulmonary regugitation correlate with the MPAP and end-diastolic pressures of pulmonary artery.Pulmonary vascular resistance (PVR) can be estimated by dividing the tricuspid regurgitation rate (TRV) (in meters per richer) by the velocity-time integral of the RVOT (in centimeters). The rationalization of this method is based on the fact that pulmonary vascular resistance is directly related to changes in pressure and inversely related to pulmonary flow. This approach has the utility of differentiating high pulmonary artery pressures caused by increased pulmonary blood flow (as occurs in hyperthyroidism, anemia, and obesity) from pulmonary hypertension due to increased PVR. Estimated pulmonary vascular resistance (PVR) may also be useful to identify patients with clinically worsening severe pulmonary hypertension without a change or decrease in MPAP as a consequence of a progressive decrease in right ventricular ejection fraction and stroke volume (PVR = MPAP-PAOP / CO. ). The tricuspid regurgitation rate (TRV) is used to determine the right ventricular systolic pressure, which is assumed to be equal to the pulmonary artery systolic pressure (SPAP) without any obstruction to pulmonary outflow and / or pulmonary valve stenosis. This value is obtained by calculating the trans tricuspid systolic gradient using the modified bernoulli equation then adding the context of the clinical setting and looking for other echocardiographic signs of excess pressure. In this case,

1. Pulmonary hypertension is unlikely with TRV $\leq 2.8 \mathrm{~m} / \mathrm{sec}, \mathrm{SPAP} \leq 36 \mathrm{mmHg}$ (assuming a RAP of $5 \mathrm{mmHg}$ ), and no echocardiographic signs suggesting pulmonary hypertension. 
2. Pulmonary hypertension is possible with $\mathrm{TRV} \leq 2.8 \mathrm{~m} / \mathrm{sec}$ and $\mathrm{SPAP} \leq 36 \mathrm{mmHg}$ but there were echocardiographic signs of pulmonary hypertension or TRV of 2.9 to $3.4 \mathrm{~m} \mathrm{/} \mathrm{sec} \mathrm{and} \mathrm{SPAP} \mathrm{of} 37$ to $50 \mathrm{mmHg}$ with or without additional signs of pulmonary hypertension.

3. Pulmonary hypertension is possible with TRV $\geq 3.4 \mathrm{~m}$ ? Seconds and SPAP $\geq 50$ $\mathrm{mmHg}$ with or without additional signs of pulmonary hypertension.

Chronic increase in right ventricular pressure, at the time of diagnosis, most patients present with echocardiography that shows enlargement of the right ventricle, right ventricular hypertrophy, increased thickness of the interventricular septum, ratio of the interventricular / posteror septum to posterior wall of the left ventricle is abnormal (> 1), and a global decline in right ventricular systolic function. In addition, the abnormal pressure gradient between the left and right ventricles results in distorted shape and displacement of the interventricular septum (flattening), which persists throughout the cardiac cycle.4 As a result, the left ventricle appears to be a D-shaped, with reduced systolic and diastolic volumes while maintaining function. global systolic. 1 Pericardial effusion and mitral valve prolapse are also seen in patients with pulmonary hypertension. Pericardial effusion may be a manifestation of impaired venous and lymphatic drainage due to an increase in right atrial pressure, and mitral valve prolapse is associated with small left ventricle and possible involvement of valve leaflets affected by associated connective tissue disorders.

At the time of definitive diagnosis, most patients with pulmonary hypertension show moderate tricuspid regurgitation with SPAP. $\geq 60 \mathrm{mmHg}$. Tricuspid regurgitation is usually caused by a widening of the tricuspid annulus, a change in the RV geometry, and a change in the apical position of the tricuspid leaflet.

\begin{tabular}{|c|c|c|}
\hline Definition & Characteristics & Clinical group $(s)^{*}$ \\
\hline $\mathrm{PH}$ & MPAP $\geq 25 \mathrm{~mm} \mathrm{Hg}$ & All \\
\hline \multirow[t]{4}{*}{ Precapillary PH } & MPAP $\geq 25 \mathrm{~mm} \mathrm{Hg}$ & 1. PAH \\
\hline & $P C W P \leq 15 \mathrm{~mm} \mathrm{Hg}$ & 3. $\mathrm{PH}$ due to lung disease \\
\hline & $\mathrm{CO}$ normal or reduced ${ }^{\dagger}$ & 4. Chronic thromboembolic $\mathrm{PH}$ \\
\hline & & 5. $\mathrm{PH}$ with unclear and/or multifactorial mechanisms \\
\hline Postcapillary PH & MPAP $\geq 25 \mathrm{~mm} \mathrm{Hg}$ & 2. $\mathrm{PH}$ due to left-heart disease \\
\hline & PCWP > $15 \mathrm{~mm} \mathrm{Hg}$ & \\
\hline & $\mathrm{CO}$ normal or reduced ${ }^{\dagger}$ & \\
\hline Passive & $\mathrm{TPG} \leq 12 \mathrm{~mm} \mathrm{Hg}$ & \\
\hline Reactive (out of proportion) & $\mathrm{TPG}>12 \mathrm{~mm} \mathrm{Hg}$ & \\
\hline
\end{tabular}

Figure 2. Hemodynamic Overview of a Patient with Pulmonary Hypertension

Significant pulmonary regurgitation is common in pulmonary hypertension. Examination of the outflow tract of the right ventricle using a pulsed-wave doppler usually shows an acceleration time of $<100$ milliseconds, which reflects abnormal MPAP.4 Due to changes in interaction between the right and left ventricles, impaired left ventricular diastolic function is characterized by dependence of left ventricular filling on arterial contraction (Bossone E, 2013).

Pulmonary hypertension is a progressive disease with a relatively poor prognosis. The history is mixed and is largely influenced by the underlying disease, with the worst prognosis for systemic sclerosis and portopulmonary hypertension and the best prognosis for congenital heart disease (because the neonatal right ventricle is well adapted to increased PV) (McLaughlin VV, 2009). 
Prior to treatment management, patients with suspected pulmonary hypertension should get confirmation of the diagnosis regardless of the cause other pulmonary hypertension using a right heart catheter, including estimation of cardiac output using either ficks or modeled.

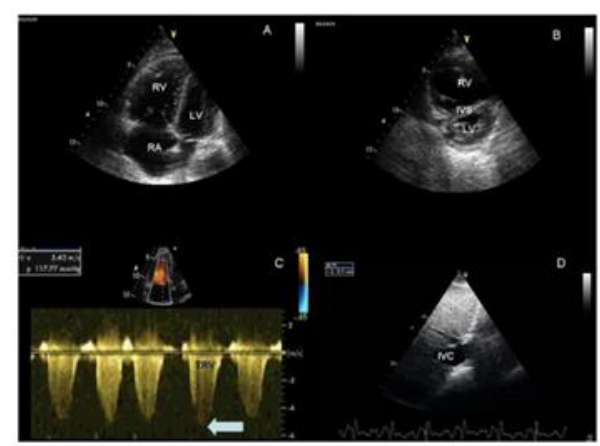

Figure 3. Display of the Four Chamber Window and Parasternal Shor Axis, Showing Severe Enlargement of The Right Ventricle and Dislocation from the Interventricular Septum (IVS) to the Left Ventricle

Although pulmonary hypertension is common in sytemic sclerosis, approximately $50 \%$ of patients with pulmonary hypertension will exhibit significant left ventricular diastolic dysfunction with increased left ventricular end-diastolic pressure. Pulmonary capillary wedge pressure (PCWP) and pulmonary artery occlusion may be inaccurate in a patient with moderate pulmonary hypertension. A careful and comprehensive prognostic evaluation in a patient with pulmonary hypertension should include the physical status using the functional classification class WHO I-IV. Exercise capacity as measured by a standard 6-minute walk or cardiopulmonary exercise test, biomarkers such as type B natriuretic peptides, kidney function, Doppler echocardiography and possibly magnetic resonance imaging. Each of these tests can correlate with findings on cardiac catheterization. All of these parameters are indicators of significant impaired right heart function and are associated with unfavorable outcomes (death or lung transplant) in patients with pulmonary hypertension (Galie N, 2009).

Predictors of prognosis using echocardiography include pericardial effusion, changes in the right atrial area, degree of displacement of the septum to the left ventricle during the diastolic period, tricuspid annular plane systolic excursion (TAPSE), pulmonary vascular capacitance, and the right ventricular doppler index (Tei index or myocardial RV. performance index). Measurement of global right ventricular function is a strong predictor of poor prognosis for pulmonary hypertension (Yeo TC, 1998).

The degree of accuracy of conventional echocardiography in depicting the structure and function of the right ventricle is subjected to several difficulties, such as the asymmetric and complex pyramidal shape of the right ventricle, plus its location on the retrosternal and endocardial surface limitations. Recent ultrasound techniques, namely tissue imaging using doppler, and three-dimensional echocardiography (RT3DE) can provide additional information in diagnosing pulmonary hypertension (Rudski LG, 2010).

Doppler tissue imaging of the mitral and tricuspid annulus has been widely applied to assess left ventricular and right ventricular function in a variety of heart diseases, including coronary heart disease, heart failure and pulmonary hypertension. 
Based on American echocardiographic society guideline data on right heart assessment, a basal right ventricular free wall value of $S{ }^{\prime}<10 \mathrm{~cm} / \mathrm{s}$ should be considered a marker of impaired right ventricular function, particularly in young adult patients (Rudski LG, 2010).

In recent years, pulmonary hypertension treatment has undergone a remarkable evolution. Recent drugs have led to a significant increase in clinical patient improvement and a reduction in the number of patients experiencing worsening. However, pulmonary hypertension is still a chronic disease for which no cure has been found. In addition, medical management and intervention for advanced cases is still invasive and susceptible to the development of significant side effects. (Galie N, 2009).

Management of patients with pulmonary hypertension cannot be considered to be limited to prescribing drugs but must be carried out in the presence of a complex strategy that includes evaluation of disease severity, supportive and general measures, assessment of vasoreactivity, estimation of success, and the combination of several different drugs plus medical intervention. At each of these stages, knowledge and experience of the medical personnel on duty is very important in maximizing existing resources (Galie $\mathrm{N}$, 2009). Patient was intubated and admitted to the intensive care unit (ICU).

Focus Assessed Transthoracic Echocardiography was performed in the ICU, where IVC enlargement was found, along with regurgitated tricuspid with pressure gradient of $33.5 \mathrm{mmHg}$ and enlargement of right ventricle. The right heart failure due to pulmonary hypertension was assessed and ventilatory support was given, along with broad spectrum antibiotics, inodilator with milrinone titrated from $0.375-0.5$ mcg.KgBW-1.min- 1 , thromboprophylaxis and other supportive care The third day of ICU care, the patient shows significant improvement. She was extubated, with no increasing work of breathing and the liver no longer palpated. After close monitoring and observation for 12 hours, the patient the stepped down to the High Care Unit (HCU). $5 \mathrm{mmHg}$ and enlargement of right ventricle.

The right heart failure due to pulmonary hypertension was assessed and ventilatory support was given, along with broad spectrum antibiotics, inodilator with milrinone titrated from 0.375 - 0.5 mcg.KgBW-1.min-1, thromboprophylaxis and other supportive care The third day of ICU care, the patient shows significant improvement. She was extubated, with no increasing work of breathing and the liver no longer palpated. After close monitoring and observation for 12 hours, the patient the stepped down to the High Care Unit (HCU). 5 $\mathrm{mmHg}$ and enlargement of right ventricle.

The right heart failure due to pulmonary hypertension was assessed and ventilatory support was given, along with broad spectrum antibiotics, inodilator with milrinone titrated from 0.375 - 0.5 mcg.KgBW-1.min-1, thromboprophylaxis and other supportive care The third day of ICU care, the patient shows significant improvement. She was extubated, with no increasing work of breathing and the liver no longer palpated. After close monitoring and observation for 12 hours, the patient the stepped down to the High Care Unit (HCU). thromboprophylaxis and other supportive care The third day of ICU care, the patient shows significant improvement. She was extubated, with no increasing work of breathing and the liver no longer palpated. After close monitoring and observation for 12 hours, the patient the stepped down to the High Care Unit (HCU). thromboprophylaxis and other supportive care The third day of ICU care, the patient shows significant improvement. She was extubated, with no increasing work of breathing and the liver no longer palpated. After close monitoring and observation for 12 hours, the patient the stepped down to the High Care Unit (HCU). 


\section{Conclusion}

The use of echocardiography in the management of critically ill patients with suspected pulmonary hypertension has become a useful and reliable modality in acute, lifethreatening situations. Although the use of a right ventricular catheter (swans-ganz catheter) remains the gold standard in enforcing pulmonary hypertension, echocardiography has the advantage that it can be done bedside, is not invasive, is faster and can be interpreted so that patient management can be carried out quickly and accurately.

\section{References}

Abbas AE, Fortuin FD, Schiller NB, Appleton CP, Moreno CA, Lester SJ. Echocardiographic determination of mean pulmonary artery pressure. Am J Cardiol. 2003 Dec 1; 92 (11): 1373-6.

Authors / Task Force Members, Galie N, Hoeper MM, Humbert M, Torbicki A, Vachiery $\mathrm{JL}$, et al. Guidelines for the diagnosis and treatment of pulmonary hypertension: The Task Force for the Diagnosis and Treatment of Pulmonary Hypertension of the European Society of Cardiology (ESC) and the European Respiratory Society (ERS), endorsed by the International Society of Heart and Lung Transplantation ( ISHLT). Eur Heart J. 2009 Oct 2; 30 (20): 2493-537.

Bossone E, D'Andrea A, D'Alto M, Citro R, Argiento P, Ferrara F, et al. Echocardiography in Pulmonary Arterial Hypertension: from Diagnosis to Prognosis. $\mathrm{J}$ Am Soc Echocardiogr. 2013 Jan; 26 (1): 1-14.

Grapsa J, Dawson D, Nihoyannopoulos P. Assessment of right ventricular structure and function in pulmonary hypertension. J Cardiovasc Ultrasound. 2011 Sep; 19 (3): $115-25$.

Mertens LL, Friedberg MK. Imaging the right ventricle - current state of the art. Nat Rev Cardiol. 2010 Oct; 7 (10): 551-63.

Rudski LG, Lai WW, Afilalo J, Hua L, Handschumacher MD, Chandrasekaran K, et al. Guidelines for the Echocardiographic Assessment of the Right Heart in Adults: A Report from the American Society of Echocardiography. J Am Soc Echocardiogr. 2010 Jul; 23 (7): 685-713.

Sangy, S. (2020). The Effects of Gavage Extract of Papaver Rhoeas L on Blood Pressure of MIA Induced Osteoarthritis and Its Interaction with Nitrergic System in male Rat. Budapest International Research in Exact Sciences (BirEx) Journal Vol 2 (4): 458466.

Sari, A.P., Lubis, R., and Zulfendri. (2020). Determinant of Hypertension, Obesity and Smoking on Stroke Events in Hospital Patients atRegional General Hospitalof Rokan Hulu Riau District, 2018(Epidemiology and Public Health). Britain International of Exact Sciences (BIoEx) Journal Vol. 2 (1):384-389.

Syahrul, et al. (2020). The Clinical Characteristics of Patients with Embolic Strokes among Indonesian Subject. Britain International of Exact Sciences (BIoEx) Journal Vol. 2 (3): 595602.

Writing Committee Members, McLaughlin VV, Archer SL, Badesch DB, Barst RJ, Farber HW, et al. ACCF / AHA 2009 Expert Consensus Document on Pulmonary Hypertension: A Report of the American College of Cardiology Foundation Task Force on Expert Consensus Documents and the American Heart Association: 
Developed in Collaboration With the American College of Chest Physicians, American Thoracic Society, Inc., and the Pulmonary Hypertension Association. Circulation. 2009 Apr 28; 119 (16): 2250-94.

Yeo TC, Dujardin KS, Tei C, Mahoney DW, McGoon MD, Seward JB. Value of a Doppler-derived index combining systolic and diastolic time intervals in predicting outcome in primary pulmonary hypertension. Am J Cardiol. 1998 May 1; 81 (9): $1157-61$. 